Originales

\title{
Tratamiento de la neutropenia febril secundaria a quimioterapia, con antibióticos orales de modo ambulatorio
}

\author{
F. Marcos Sánchez, I. Albo Castaño, F. Árbol Linde, A. Viana Alonso, F. Juárez Ucelay, A. Durán Pérez-Navarro
}

\section{Resumen}

- Propósito: analizar si el tratamiento con antibióticos por vía oral de modo ambulatorio, resulta eficaz en pacientes con neutropenia febril de bajo riesgo.

- Material y métodos: estudio observacional, prospectivo de casos clínicos. 10 pacientes incluídos, que cumplían los criterios de neutropenia febril de bajo riesgo, recibieron tratamiento antibiótico con quinolonas, ciprofloxacino en ocho casos y en los otros dos levofloxacino, durante un periodo de 10 a 14 días. En los tres primeros casos incluídos cronológicamente se asoció tratamiento con filgastrim. Se efectuaron revisiones clínicas a las 48 horas y posteriormente cada 48-72 horas, hasta la resolución de la neutropenia febril.

- Resultados: la fiebre desapareció a las 48 horas en cinco casos, a las 72 horas en otros tres enfermos y persistió febrícula en dos casos, que estimamos secundaria al cáncer y no a la neutropenia. Un nuevo control hematológico a las 48 horas, mostró un recuento de neutrófilos superior a 500 en tres casos y a los 4 días del episodio inicial, cinco de los 6 casos restantes tuvieron un recuento de neutrófilos superior a 500. Ningún paciente precisó ingreso hospitalario y como medida de precaución adicional, decidimos retrasar una semana y reducir al $25 \%$ la siguiente administración de quimioterapia.

- Conclusiones: el tratamiento con antibióticos por vía oral de modo ambulatorio resulta eficaz en los pacientes que presentan neutropenia febril de bajo riesgo, evitándose ingresos innecesarios y complicaciones hospitalarias.

Palabras claves:

Neutropenia febril. Quinolonas. Tratamiento ambulatorio.

Oncología, 2004; 27 (9):533-536 


\section{Summary}

- Purpose: To analyze whether oral antibiotic therapy is effective for patients under outpatient regime having a low risk febrile neutropenia.

- Material and methods: An observational, prospective study of 10 patients meeting the criteria of low risk neutropenia is made. Quinolones were administered, emplpying ciprofloxacin in 8 cases and levofloxacin in the other 2 during 10-14 days. The first three cases seen received also filgastrim. The patients were followed-up after 24 hours, and then every 48-72 hours until the febrile neutropenia resolved.

- Results: Fever disappeared within $\mathbf{4 8}$ hours in 5 cases and within $\mathbf{7 2}$ hours in 3 patients. A low grade fever remained in 2 cases, that according to the authors was cancer-related and not caused by neutropenia. The hematological control showed a neutrophil count over 500 per mm 3 after $\mathbf{4 8}$ hours in 3 cases, and 4 days after the beginning of the episode a count over 500 was found in five out of the six remaining patients. No patient required hospitalization. As an additional preventive measure, the next chemotherapy course was delayed one week and the dose reduced to $25 \%$ of the total dose.

- Conclusions: Oral antibiotic therapy under outpatient regime is effective for patients with lowrisk febrile neutropenia, thus avoiding unnecessary hospital admissions and complications.

Key words: Febrile neutropenia. Quinolones. Outpatient therapy.

\section{Introducción}

La neutropenia esta considerada como un importante factor favorecedor de infecciones en los enfermos que reciben quimioterapia para el tratamiento de diversas neoplasias.

En los pacientes con neutropenia febril, la hospitalización precoz y el tratamiento intravenoso con antibióticos de amplio espectro, ha mostrado una importante reducción en el número de infecciones documentadas y una disminución significativa en la mortalidad de estos enfermos'. Sin embargo, hay evidentes problemas derivados de la hospitalización de los pacientes con cáncer y neutropenia febril, entre los que se incluye la toxicidad de los antimicrobianos; el aumento de los costes que implica la hospitalización; exposición a patógenos nosocomiales, en la mayoría de los casos con una mayor agresividad que los que se pueden adquirir en el medio domiciliario; pérdida de la calidad de vida, con el consiguiente deterioro psicológico y también económico al no poder realizar ningún tipo de trabajo al encontrarse hospitalizado?

Además en la mayoría de los casos la fiebre desaparece en pocos días, los diferentes cultivos extraídos son negativos, por lo que parece probable que en muchas ocasiones no fuera precisa la hospitalización de los pacientes con neutropenia febril.

Diversos estudios realizados en los últimos años avalan la posibilidad del manejo ambulatorio en una gran mayoría de éstos enfermos ${ }^{2-6}$.

Nos parece interesante presentar los resultados obtenidos con el manejo ambulatorio mediante antibióticos de amplio espectro por vía oral en pacientes con neutropenia febril de bajo riesgo, secundaria a la administración de quimioterapia.

\section{Material y métodos}

Definimos la neutropenia febril como aquella situación clínica caracterizada por un recuento de neutrófilos igual o inferior a $500 \mathrm{~mm}^{3}$. Se había objetivado una temperatura aislada de $38,5^{\circ} \mathrm{C}$ o tres tomas de $38^{\circ} \mathrm{C}$, dentro de un período de tiempo de veinticuatro horas y separadas entre sí al menos 4 horas.

Se definía como neutropenia de bajo riesgo aquella situación clínica en la que la duración anticipada de la neutropenia se estimara inferior a 7 días, el paciente presentaba un tumor sólido en tratamiento de quimioterapia, ausencia de enfermedades o situaciones médicas, tales como hipotensión arterial, deterioro del estado mental, insuficiencia respiratoria, deshidratación, hemorragia significativa, compromiso cardíaco o aparición de arritmias, infección del túnel del catéter u otra celulitis extensa, insuficiencia renal o hepatopatía crónica previa, se debía descartar la existencia de 
neumonía y de otras infecciones graves (meningitis, etc.), el paciente debía estar previamente ambulatorio $^{7,8}$.

Se incluyeron en el estudio los pacientes con neutropenia febril de bajo riesgo, excluyendo los pacientes que hubieran ingresado en el hospital durante las dos semanas previas, no debían haber recibido tratamiento con quinolonas previamente, se excluyeron asimismo los pacientes con fiebre originada por transfusión de hemoderivados, que aparece generalmente durante las 6 horas siguientes a la transfusión y los pacientes que residieran a una distancia del centro hospitalario superior a 45 minutos, mediante traslado mecanizado.

Se realizó un estudio observacional, prospectivo, de casos clínicos, de los pacientes que cumplían los criterios anteriormente referidos y que acudieron a la consulta de oncología médica de un hospital del grupo 2 en el plazo de 2 años.

Los pacientes recibieron tratamiento con antibióticos por vía oral, ciprofloxacino a dosis de 500 miligramos cada 12 horas o levofloxacino a dosis de 500 miligramos cada 24 horas, durante 10 a 14 días. Además se asoció paracetamol a dosis de 500 miligramos cada 8 horas y en los 3 primeros casos incluidos cronológicamente se asoció factor estimulante de colonias granulocíticas recombinante (filgastrim), a dosis de $300 \mathrm{mi}-$ crogramos, por vía subcutánea cada 24 horas durante al menos 3 días.

Se extrajeron dos hemocultivos el primer día y se realizó una radiografía de tórax en la que no se observaba una condensación neumónica.

Se revisaron los pacientes en las consultas externas a las 48 horas, realizándose un control clínico y hematológico y posteriormente según los casos, se realizaron otras revisiones cada 48-72 horas, hasta constatarse la desaparición de la neutropenia y de la fiebre.

\section{Resultados}

10 pacientes incluidos, 7 mujeres y 3 hombres, de edades comprendidas entre 48 y 73 años, con una edad media de 63 años.

Recibían tratamiento de quimioterapia por una neoplasia de mama 6 casos, 2 cáncer de pulmón y otros dos cáncer de colon o recto.

Se diagnóstico neutropenia febril a los 7 días de la administración de la quimioterapia en un caso, de 8 días en tres casos, en el día noveno en otros cuatro casos, en el día décimo en dos enfermos.

En el estudio hematológico se objetivaron 200 neutrófilos $/ \mathrm{mm}^{3}$ en cuatro casos, 300 neutrófilos en otros cuatro casos y 400 neutrófilos en dos pacientes.
Se administró tratamiento con ciprofloxacino a dosis de 500 miligramos cada 12 horas, por vía oral, en ocho enfermos, levofloxacino a dosis de 500 miligramos por vía oral en dos casos.

En tres enfermos se administró filgastrim a dosis de 300 microgramos al día por vía subcutánea, en dos casos durante 3 días y en otro durante 5 días.

Se realizó un control hematológico y clínico a las 48 horas y en tres casos el recuento de neutrófilos fue entonces ya superior a $500 / \mathrm{mm}^{3}$, y en los restantes casos había ascendido respecto al valor previo. En el siguiente control realizado a los 4 días del episodio inicial, cinco de seis casos mostraron ya un recuento de neutrófilos superior a $500 / \mathrm{mm}^{3}$ y en otro enfermo se realizó el segundo control a los 5 días del diagnóstico de la neutropenia febril y dicho recuento de neutrófilos fue también superior a $500 / \mathrm{mm}^{3}$.

Desapareció la fiebre a las 48 horas del diagnóstico en cinco casos, a las 72 horas en otros 3 casos y en otro dos persistió temperatura inferior a $38^{\circ} \mathrm{C}$, que estimamos secundaria al cáncer y no a la neutropenia.

No se observó crecimiento de microorganismos en ninguno de los hemocultivos extraídos.

Ningún paciente precisó ingreso hospitalario y la evolución posterior fue favorable.

Como medida de precaución retrasamos la siguiente administración de quimioterapia y reducimos asimismo la dosis al $25 \%$ de la inicial.

\section{Discusión}

La neutropenia febril, constituye una situación clínica de potencial gravedad, que requiere una rápida identificación, un manejo protocolizado y controlado y un tratamiento antibiótico adecuado y precoz.

La intensidad y duración de la neutropenia constituye el principal factor de riesgo para la adquisición de infecciones bacterianas y por hongos.

En los últimos años se ha reconocido que los pacientes con neutropenia febril constituyen un grupo de población muy heterogéneo, con riesgos muy diferentes para el desarrollo de infecciones graves durante el episodio y por este motivo su manejo clínico se ha modificado de modo notable recientemente $e^{2-5,7,8}$.

Los antibióticos que se empleen deben tener una gran actividad sobre microorganismos gram positivos y negativos, aunque en la mayoría de los estudios los gérmenes gram negativos han sido los más frecuentemente encontrados?.

Un posible problema que podíamos encontrar por la administración de quinolonas, era un incremento de resistencias de los gérmenes gram negativos a estos 


\section{F. Marcos Sánchez y cols.}

antimicrobianos, evidenciado en algunos estudios 10,11 . Este problema tratamos de reducirlo excluyendo del tratamiento por vía oral de modo ambulatorio a los pacientes que hubieran recibido tratamiento previo con quinolonas.

Aunque inicialmente se excluían del tratamiento antibiótico ambulatorio los pacientes de más de 65 años de edad, posteriormente se incluyeron en los programas de tratamiento domiciliario de la neutropenia febril en pacientes de bajo riesgo ${ }^{12}$.

Resulta evidente que la mayoría de los pacientes con cáncer y neutropenia febril, prefiere realizar un tratamiento en su domicilio, teniendo en cuenta la mayor calidad de vida que dicho tratamiento condiciona ${ }^{12}$.

Dado que uno de los factores pronóstico más importante es la duración de la neutropenia, resulta prudente incluir únicamente enfermos con una duración estimada inferior a siete días, por lo que excluimos los pacientes con neutropenia febril en el curso de un tratamiento de leucemia o que habían recibido transplantes de médula ósea en otro centro, situaciones éstas en la que la neutropenia febril es bastante más frecuente y grave ${ }^{5}$.

El tratamiento con factores estimulantes de colonias granulocíticas ha demostrado una reducción en la duración de la neutropenia, pudiendo acortarse la estancia hospitalaria de un eventual ingreso, así como el tiempo del tratamiento antibiótico ${ }^{13}$. Sin embargo, estudios recientes no han demostrado una disminución de la mortalidad, razón por la cuál ya no son empleados dichos fármacos de modo rutinario en los pacientes con neutropenias febriles de bajo riesgo.

Resulta muy importante individualizar cada caso, analizando la posible duración de la neutropenia febril teniendo en cuenta el día de la administración del ciclo de quimioterapia, estimar la posible reserva medular revisando los tratamientos de quimioterapia que hubiera recibido con anterioridad y las cifras de neutrófilos con las que hemos partido en el ciclo actual; asimismo debemos explorar exhaustivamente al paciente, valorando su estado físico, la existencia de otras enfermedades y debemos tener en cuenta la opinión del paciente sobre la posible aceptación de un tratamiento ambulatorio con un control muy exhaustivo de su evolución, explicándole que la neutropenia febril es una complicación potencialmente grave de la quimioterapia en pacientes con cáncer y posteriormente debemos analizar todos los datos recogidos para actuar convenientemente.

Correspondencia:

Dr. F. Marcos Sánchez

Escosura, 4 - 6음

E-28015 Madrid

\section{Bibliografía}

1. Hughes WT, Armstrong D, Bodey GP, Feld R, Mandell GL, Mayer JD, et al. Guidelines for the use of antimicrobial agents in neutropenic patients with unexplained fever. Clin Infect Dis 1997; 25:551-3.

2. Hidalgo M, Hornedo J, Lumbreras C, Trigo JM, Colomer $\mathrm{R}$, Perea $\mathrm{S}$, et al. Outpatient therapy with oral Ofloxacin for patients with low risk neutropenia and fever. Cancer 1999; 85:213-9.

3. Noseda MA, Cipri A, De Marinis F, Migliorino MR, Gareri $R$, Bigioni $D$, et al. Ciprofloxacin and G-CSF for fever treatment in neutropenic outpatients with advanced non small lung cancer (NSCLC), treated with chemotherapy (CT). Lung Cancer 1997; 18:207.

4. Rubenstein EB, Rolston K, Benjamin RS, Loewy J, Escalante C, Manzullo E, et al. Outpatient treatment of febrile episodes in low- risk neutropenic patients with cancer. Cancer 1993; 71:3640-6.

5. Malik IA, Wajahat AK, Karim M, Aziz Z, Ata Khan M. Feasibility of outpatient management of fever in cancer patients with low-risk neutropenia: results of a prospective randomized trial. Am J Med 1995; 98:224-31.

6. Rubenstein EB, Rolston KVI. Outpatient treatment of febrile neutropenic patients with cancer. Eur J Cancer 1995; $31: 2-4$.

7. Talcott JA, Siegel RD, Finberg R, Goldman L. Risk asessment in cancer patients with fever and neutropenia: a prospective, two center validation of a prediction rule. J Clin Oncol 1992; 10:316-22.

8. Talcott JA, Finberg R, Mayer RJ, Goldman L. The medical course of cancer patients with fever and neutropenia: clinical identification of a low-risk subgroup at presentation. Arch Intern Med 1988; 144:2561-8.

9. EORTC International Antimicrobial Therapy Cooperative Group and the National Cancer Institute of Canada-Clinical Trials Group. Vancomycin added to empirical combination antibiotic therapy for fever in granulocytopenic cancer patients. J Infect Dis 1991; 163:951-8.

10. Carratalá J, Fernández-Sevilla A, Tubau F, Domínguez MA, Gudiol F. Emergence of quinolone- resistant Escherichia coli bacteremia in neutropenic patients with cancer who have received prophylactic norfloxacin. Clin Infect Dis 1995; 20:557-60.

11. Kern WV, Andriof E, Oethinger M, Kern P, Hacker J, Marre R. Emergence of quinolone - resistant Escherichia coli at a cancer center. Antimicrob Agents Chemother 1994; 38:681-7.

12. Talcott JA, Whalen A, Clark J, Rieker PP, Finberg R. Home antibiotic therapy for low-risk cancer patients with fever and neutropenia: a pilot study of 30 patients based on a validated prediction rule. J Clin Oncol 1994; 12: 107-14.

13. Molina F. Complicaciones infecciosas en los pacientes con cáncer. Oncología médica, de Rosell R, Abad A, Monzo M, Molina F. Ediciones Ergón. Madrid 1995; 383-99. 